\title{
A role for the histone deacetylase HDAC4 in the life-cycle of HIV-1-based vectors
}

\author{
Johanna A Smith", Jennifer Yeung ${ }^{1}$, Gary D Kao², René Daniel ${ }^{1,3,4^{*}}$
}

\begin{abstract}
HIV-1 integration is mediated by the HIV-1 integrase protein, which joins 3'-ends of viral DNA to host cell DNA. To complete the integration process, HIV-1 DNA has to be joined to host cell DNA also at the $5^{\prime}$-ends. This process is called post-integration repair (PIR). Integration and PIR involve a number of cellular co-factors. These proteins exhibit different degrees of involvement in integration and/or PIR. Some are required for efficient integration or PIR. On the other hand, some reduce the efficiency of integration. Finally, some are involved in integration site selection. We have studied the role of the histone deacetylase HDAC4 in these processes. HDAC4 was demonstrated to play a role in both cellular double-strand DNA break repair and transcriptional regulation. We observed that HDAC4 associates with viral DNA in an integrase-dependent manner. Moreover, infection with HIV-1-based vectors induces foci of the HDAC4 protein. The related histone deacetylases, HDAC2 and HDAC6, failed to associate with viral DNA after infection. These data suggest that HDAC4 accumulates at integration sites. Finally, overexpression studies with HDAC4 mutants suggest that HDAC4 may be required for efficient transduction by HIV-1-based vectors in cells that are deficient in other DNA repair proteins. We conclude that HDAC4 is likely involved in PIR.
\end{abstract}

\section{Introduction}

Chromatin undergoes expansion and compaction in the course of many fundamental cellular processes, including gene expression, differentiation, cell cycle progression and DNA repair. These alterations of the chromatin structure are largely mediated by histone acetylases and histone deacetylases (HDACs). HDACs deacetylate key lysine residues of core histones to induce chromatin compaction. This process usually results in transcriptional repression [1]. Cells contain many HDACs, which are categorized into four classes, based on sequence homologies. Class I (homologues of the yeast deacetylase Rpd3) contains HDAC1, HDAC2, HDAC3 and HDAC8 [2-6]. Class II (yeast Hda1 homologues) contains HDAC4, HDAC5, HDAC6 and HDAC7 [7-12]. Class II HDACs, unlike Class I, can shuttle in and out of the nucleus, depending on various signals [13]. Class III contains proteins that are homologous to the yeast deacetylase Sir $2[14,15]$. Finally, the Class IV contains enzymes which are related to those of Class I and Class II, but a

\footnotetext{
* Correspondence: Rene.Daniel@jefferson.edu

'Division of Infectious Diseases - Center for Human Virology, Department of Medicine, Thomas Jefferson University, Philadelphia, PA 19107, USA Full list of author information is available at the end of the article
}

sequence analysis shows they form a distinct class. They are exemplified by HDAC11 [16].

Although transcriptional repression is apparently an important function of HDACs, these proteins seem to play a broader role in regulating cellular processes and one HDAC, HDAC4, has been found to play a role in cellular double-strand DNA break (DSB) repair. It has been shown by Kao et al. (2003) that HDAC4 forms nuclear foci in cells exposed to ionizing radiation, which causes double-strand DNA breaks [17]. Foci of DNA repair proteins are formed at sites of double-strand DNA breaks, and the HDAC4 foci overlap with foci of the DNA repair proteins Rad51 and 53BP1. Silencing of HDAC4 via RNA interference leads to radiosensitisation of HeLa cells, underscoring a requirement for HDAC4 in DSB repair. In addition, HDAC4-deficient cells were shown to loose the DNA damage-induced G2/M checkpoint. The molecular function of HDAC4 in DSB repair remains to be fully clarified, although it has been shown very recently that nuclear translocation of HDAC4 is required and it may play a role in the suppression of promoters of genes that are activated during G2/M progression $[18,19]$.

It has been shown previously by us and others that cellular DSB repair proteins are involved in the life-cycle of retroviruses and retroviral vectors. We have observed

C Biomed Central

C 2010 Smith et al; licensee BioMed Central Ltd. This is an Open Access article distributed under the terms of the Creative Commons Attribution License (http://creativecommons.org/licenses/by/2.0), which permits unrestricted use, distribution, and reproduction in any medium, provided the original work is properly cited. 
that cellular DSB proteins are involved in completing the integration process. In addition, others suggested that they are involved in the formation of 2-LTR circles, and it has been proposed that they might also be involved in intranuclear trafficking of the preintegration complex [20-23].

In this study, we have tested the hypothesis that HDAC4 plays a role in the life-cycle of HIV-1-based vectors. We show that infection with retroviral vectors induces, similar to DSBs, nuclear foci of the HDAC4 protein. We show that the formation of these foci is dependent on active retroviral integrase, and HDAC4, but not HDAC2 and HDAC6, associates with viral DNA. Taken together, these data indicate that HDAC4 plays a yet undiscovered role at sites of retroviral DNA integration. In addition, we show that overexpression of nuclear HDAC4 rescues a defect in retroviral transduction that is associated with a deficiency of the cellular DNA repair protein ATM. We conclude that HDAC4 is involved in stable transduction by retroviral vectors, and plays a role in the completion of the integration process.

\section{Results \\ HDAC4, but not HDAC2 or HDAC6, associates with DNA of an infecting HIV-1-based vector}

HeLa cells were infected with a pseudotyped HIV-1based vector (containing a lac Z reporter) at an m.o.i. of 0.1 and harvested at the time points indicated (Fig. 1A). Chromatin immunoprecipitation (ChIP) analysis was used to identify the association of HDAC4 with viral DNA. To do so, DNA isolated from infected cells and the associated proteins were crosslinked, immunoprecipitated with the HDAC4 antibody (see Methods), and associated viral DNA was amplified by real time PCR. Results are expressed as a number of viral DNA amplicons per $\mu$ l of chromatin immunoprecipitates at each time point. As shown in Fig. 1A, viral DNA was found to be associated with HDAC4 at 4, 6, 8, and $16 \mathrm{hrs}$ post-infection. The amount of HDAC4-associated viral DNA steadily increased from 4 hours, with a peak reached at 8 hours post-infection. The associated viral DNA drastically declined at the 16 hour time point. To determine if vector DNA associates with other HDAC proteins, we have immunoprecipitated lysates from infected cells with the HDAC2 and HDAC6 antibodies. Whereas HDAC2 is a Class I HDAC, we note HDAC6 is a class II HDAC and thus structurally closely related to HDAC4. However, as shown in Fig. 1B, we did not observe any association of these HDACs with viral DNA. We thus conclude that HDAC4 shows a distinct preference for association with vector DNA, when compared to other HDACs.
Retroviral integration enhances the association of HDAC4 with vector DNA

We note that HDAC4 was reported to associate with DNA of the avian sarcoma virus, but this association was detected only post-integration [24]. To test the hypothesis that integration is required for the association of HDAC4 with the DNA of HIV-1-based vectors, we have infected HeLa cells and treated them with the integrase inhibitor 118-D-24. As shown in Fig. 1C, the inhibitor decreases the association of HDAC4 with vector DNA in a dose-dependent manner. However, we note that the inhibitor effect can be seen only at 8 hours post-infection, when the association of DNA with HDAC4 is at its peak. In contrast, association at 4 hours post-infection is resistant to the inhibitor treatment. These data suggest that while integration does stimulate the association of vector DNA with HDAC4, HDAC4 also associates with vector DNA prior to integration, in an integration-independent manner.

\section{Retroviral integration induces the formation of HDAC4 foci in infected cells}

HDAC4 was reported to form foci in irradiated cells. These foci were associated with the formation of double-strand DNA breaks [17]. To determine if infection with HIV-1-based vectors induces the formation of HDAC4 foci, we have infected HeLa cells at a high multiplicity of infection (10), fixed infected cells at predetermined time points and stained with the HDAC4 antibody. We observed that in uninfected cells, HDAC4 is present almost exclusively in the cytoplasm (Fig. 2). Similarly, we have observed that HDAC4 is predominantly cytoplasmic at 4 and 6 hours post-infection. However, we also observed the appearance of HDAC4 foci in infected cells, with the majority of cells containing foci at $8 \mathrm{hrs}$ post-infection (Figs. 2 and 3). Most of the infected cells contained multiple HDAC4 foci. As indicated in Fig. 3, the number of foci correlates well with the multiplicity of infection.

We have observed that integration stimulates the association of HDAC4 with vector DNA and wondered if integration affects the formation of HDAC4 foci. Thus, we have infected HeLa cells in the presence and absence of an integrase inhibitor. We have again detected HDAC4 foci in cells that were infected with the HIV-1based vector in the absence of the inhibitor (Fig. 4). However, treatment of infected cells with the inhibitor significantly reduced (ca. 3.5 fold) the total number of cells that contained foci (Fig. 4 and Fig. 5). We have also observed a drop in the average number of foci per cell among foci-containing cells, although the difference was within the standard deviation due to a wide range of the numbers of foci ( 1 to 8 foci per cell among cells 


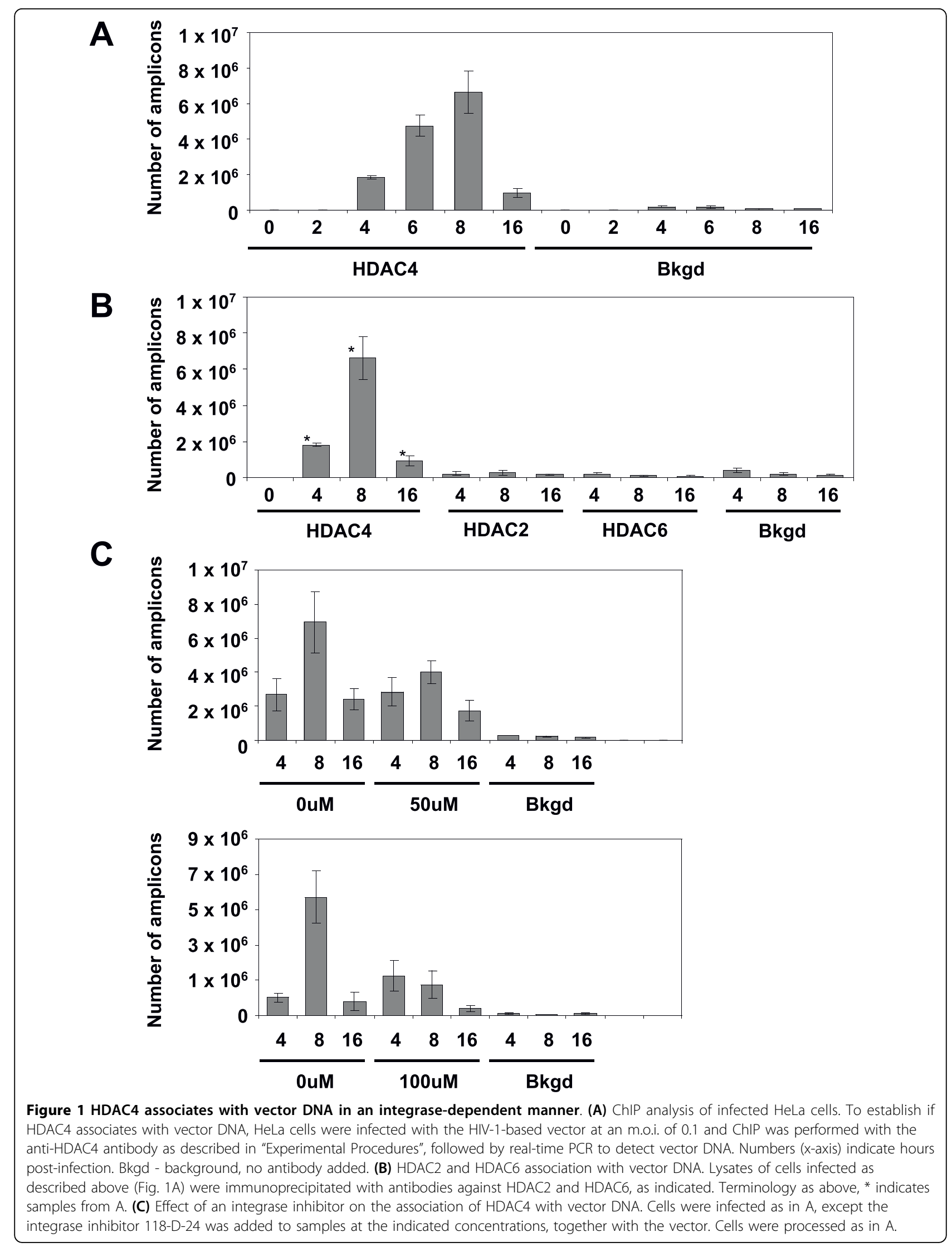




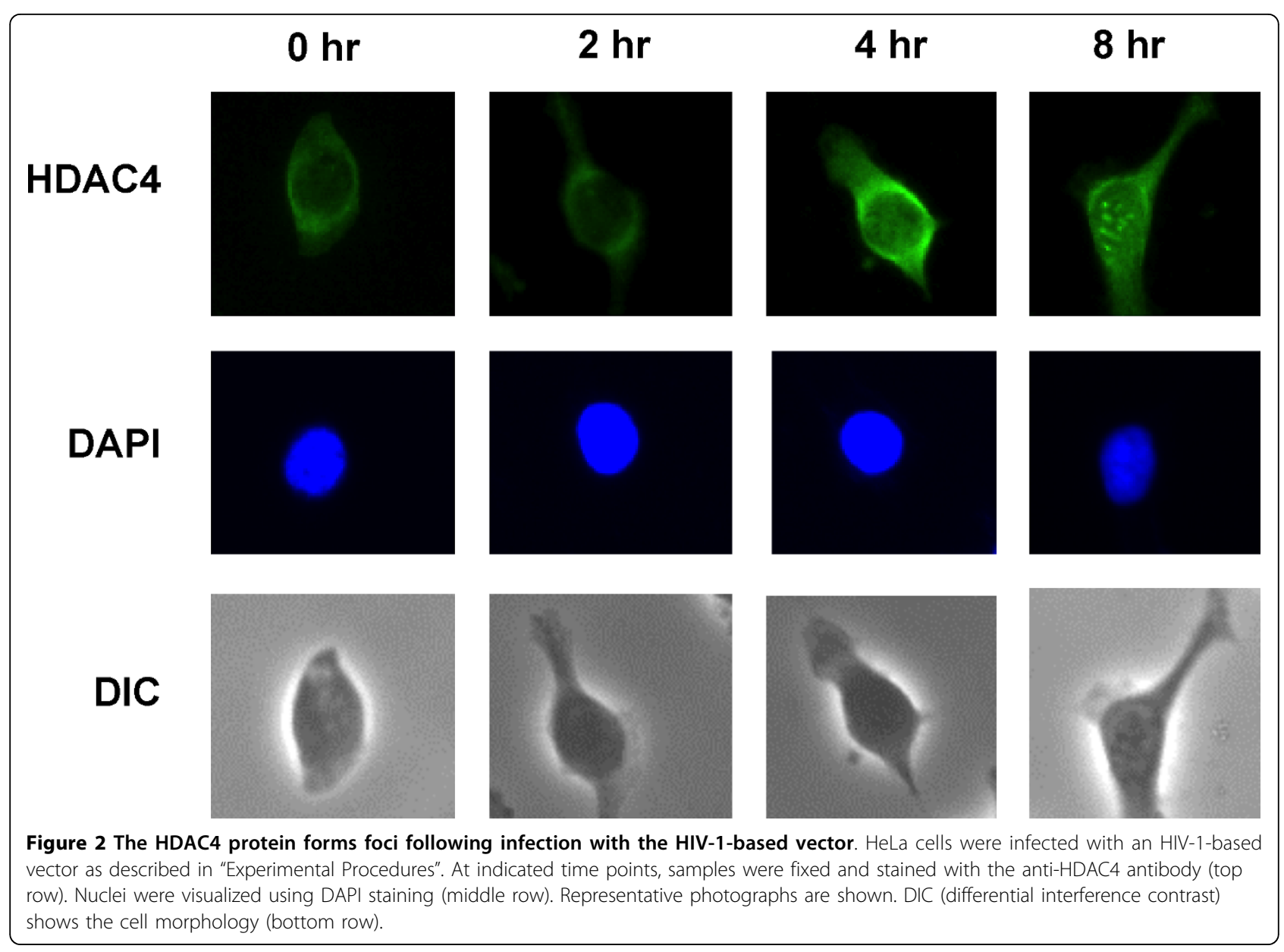

infected with the vector and 1 to 6 foci per cell in cells infected with the vector and treated with the inhibitor, Fig. 5B). Treatment with the inhibitor itself had a negligible effect on the intracellular localization of HDAC4 (Figs. 4 and 5). Taken together, our results suggest that although HDAC4 associates with viral DNA even prior to integration, integration stimulates further accumulation of HDAC4 at integration sites, which are then marked by the formation of HDAC4 foci.

\section{Effect of HDAC4 knockdown on HIV-1 transduction}

We have established a novel interaction between the cellular HDAC4 protein and HIV-1-based vectors. Our results suggested that HDAC4 plays a role in the lifecycle of these vectors. To test this hypothesis, we have knocked down HDAC4 in HeLa cells using siRNA treatment and determined if HDAC4 is required for stable integration of HIV-1- vector DNA. As shown in Fig. 6, HDAC4 had little effect on the efficiency of integration as measured by $A l u$-PCR. In addition, we have infected siRNA-treated cells with the HIV-1-based vector carrying an EGFP marker and examined EGFP expression using flow cytometry. We have not observed a significant drop in EGFP expression in HDAC4 siRNA-treated cells (data not shown). We conclude that HDAC4 deficiency does not appear to significantly affect the efficiency of integration. Similarly, it appears that HDAC4 is not necessary for the last step of the integration process, termed post-integration repair (PIR), since PIR failure results in a loss of cells in which integrase-mediated joining occurred, and thus again manifests as a decrease in the $A l u$-PCR signal $[25,26]$.

\section{HDAC4 is involved in PIR in ATM-deficient cells}

HDAC4 is a DSB repair protein, and it had been reported by us and others that these proteins are involved in PIR [23]. However, cellular DSB repair proteins often have overlapping functions and DSB repair systems can partially substitute for each other [27]. It is thus possible that a loss of the HDAC4 protein can be compensated for by other DSB repair systems or proteins. To test this hypothesis, we have induced a DSB repair deficiency in HeLa cells by treatment with an established ATM inhibitor, KU-55933 [28]. The ATM 


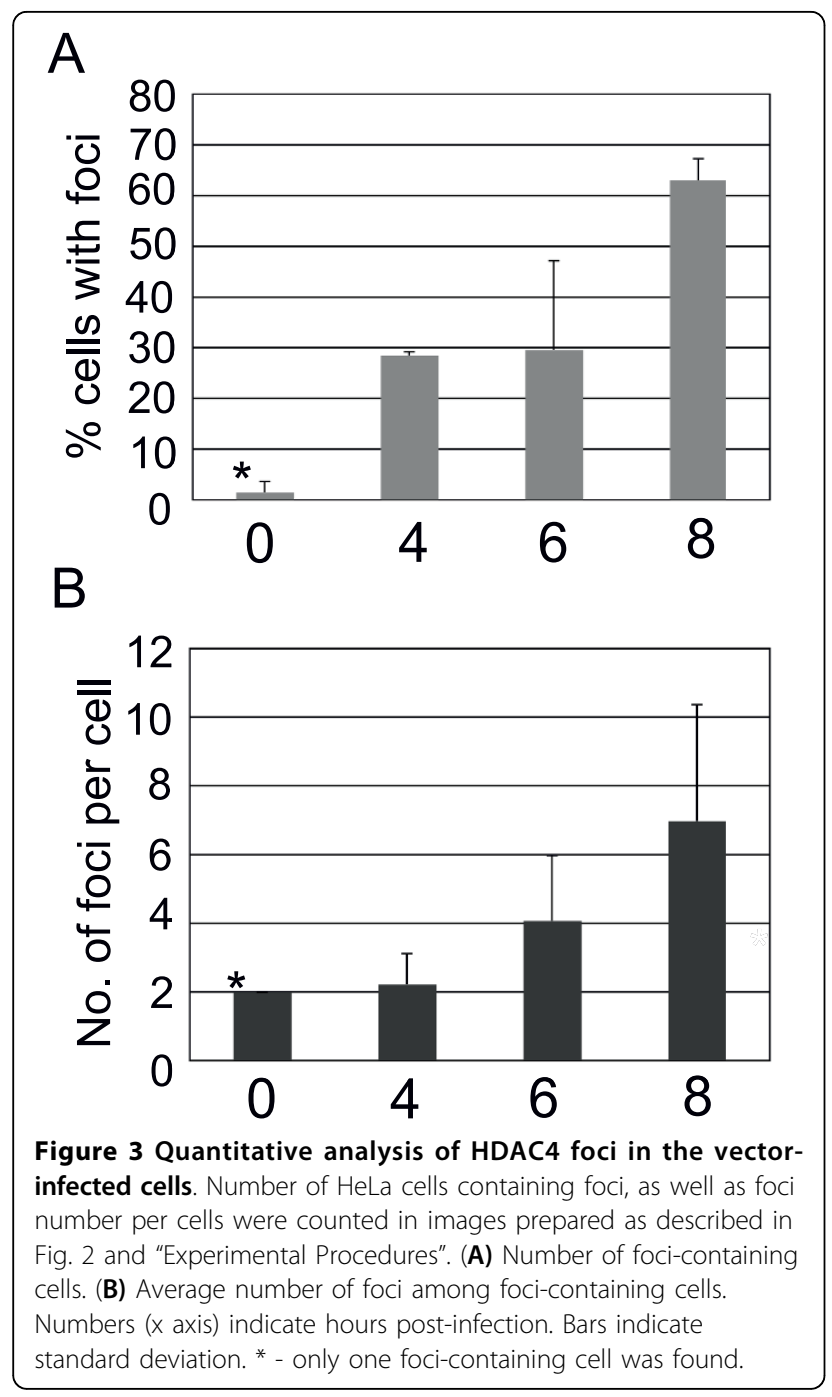

protein is a major player in cellular DSB repair and was reported by us and others to be involved in PIR [26-28]. At the same time, in these cells, we have overexpressed the HDAC4 protein. Since the normal HDAC4 protein (denoted here as HDAC4-1084) is mainly cytoplasmic, we have also overexpressed a mutant, which lacks a nuclear export signal (HDAC4-1061) and is thus present in the nucleus (Fig. 7, [29]). As expected, the ATM inhibitor reduced the $A l u$-PCR signal due to the inhibition of PIR (Fig. 7A). We have observed that in ATM-proficient HeLa cells, the overexpressed HDAC4 proteins do not appear to affect the efficiency of integration or PIR, as shown by $A l u$-PCR (Fig. 7A). However, in HeLa cells that were treated with the ATM inhibitor, the HDAC41084 reverses the inhibitor effect and upregulates HIV-1 transduction four fold. The HDAC4-1061 mutant that is constitutively present in the nucleus completely reverses the effect of the ATM inhibitor (Fig. 7A). To investigate the possibility that the differences in the Alu-PCR signals could be due to variations of the exogenous HDAC4 expression levels, we performed a western bloting analysis (Fig. 7B). However HDAC4-1061 and HDAC4-1084 levels appear to be the same in our transfected cells. Taken together our results suggest that HDAC4 is involved in PIR, but its function can be replaced by other DSB protein(s).

Finally, a failure of PIR induces apoptotic death of infected cells. If the effect of nuclear HDAC4 on infection efficiency is due its role in PIR, it should prevent PIR-associated cell death. Thus, cells were treated and infected as above (Fig. 7), except at a high m.o.i. (2), and analyzed by Western blotting for the presence of the 85kDa PARP fragment, an apoptotic marker generated by caspase-mediated cleavage of the PARP protein [30]. As shown in Fig. 8, ATM inhibition and infection stimulated PARP cleavage. However, the apoptosis was reduced by overexpression of either the full length HDAC4 (HDAC4-1084) or the truncated mutant (HDAC4-1061). This finding is again consistent with an HDAC4 role in PIR.

\section{Discussion}

In this study, we demonstrate that the histone deacetylase HDAC4, a Class II HDAC, associates with DNA of HIV-1-based vectors and forms foci at sites of integration. We also show that overexpression of nuclear HDAC4 rescues the defect in PIR that is induced by an ATM deficiency. Our data thus reveals a new cellular partner, which is involved in the life-cycle of HIV-1based vectors. Our finding also supports the hypothesis that cellular DSB repair proteins are involved in PIR. At the same time, these proteins clearly have overlapping functions and can to a degree substitute for each other.

What could be the HDAC4 function in PIR? HDAC4 is a deacetylase, although with relatively low activity [31]. Histone deacetylation generally results in transcriptional suppression. Thus, one possible function of HDAC4 could be to suppress transcription at integration sites, thus allowing access for DNA repair machinery. We note in this context that HIV-1 prefers to integrate in genes and the likelihood of transcription interfering with integration is thus very high [32]. Second, it is possible that HDAC4 is required for the recruitment of other DNA repair proteins to PIR sites. HDAC4 was reported to physically interact with the $53 \mathrm{BP} 1$ protein and thus may bring this protein to PIR sites. It will be a matter of future experiments to distinguish between these possibilities.

We also note that HDAC4 associates with vector DNA prior to integration. These data suggest that HDAC4 may play a role in steps prior to PIR. However, since HDAC4 knockdown does not appear to have a major effect on the transduction efficiency of the vector, 


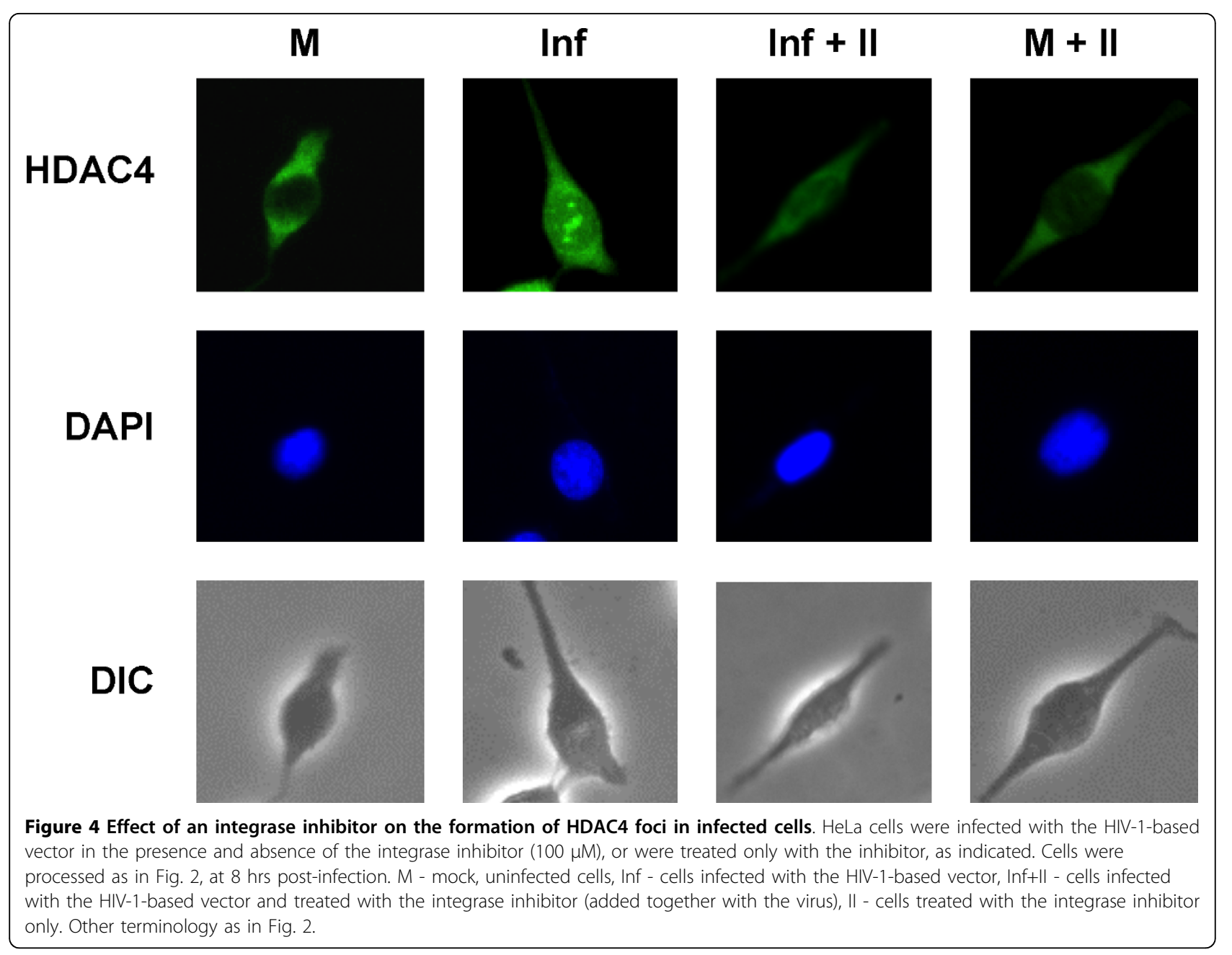

it seems likely that HDAC4 is not required for these steps of the retroviral life-cycle. Nevertheless, it is possible that HDAC4 affects the life-cycle in different ways. One possibility is that HDAC4 has a cell-type-specific function and affects the retroviral life-cycle differently depending on cell type. Another possibility is that HDAC4 affects intracellular or intranuclear trafficking, which may effect integration site selection. Experiments designed to test these hypotheses are underway in our laboratory.

What are the practical implications of our data? HIV1-based vectors perform integration and PIR in an identical way to wild-type HIV-1. Thus, proteins which are required for PIR of HIV-1-based vectors are also involved in PIR of HIV-1. Since PIR is absolutely required for HIV-1 replication, proteins involved in PIR are potential targets for ani-HIV-1-therapy. However, overlapping functions of these proteins suggest that it will be necessary to inhibit more than one DNA repair pathway to achieve complete suppression of HIV-1 replication.
Finally, our results indicate that HDAC4 accumulates at the sites of integration. HDAC4 foci thus may serve as a useful marker for integration, and their numbers could be used to evaluate the efficacy of HIV-1- inhibitors at the early steps of the HIV-1- life-cycle.

\section{Experimental Procedures \\ Cells}

HeLa cells were maintained in DMEM medium supplemented with $10 \%$ fetal bovine serum and antibiotics (Penn/Strep).

\section{HIV-1-based vectors}

All VSV G-pseudotyped HIV-1 based vectors were prepared as described previously [33,34], and carried either a lacZ or EGFP reporter gene.

\section{Plasmids and transfections}

Plasmids expressing the full-length HDAC4 (amino acids 1-1084) fused to the EGFP protein (HDAC4-1084) or the HDAC4 C-terminal truncated mutant, lacking 


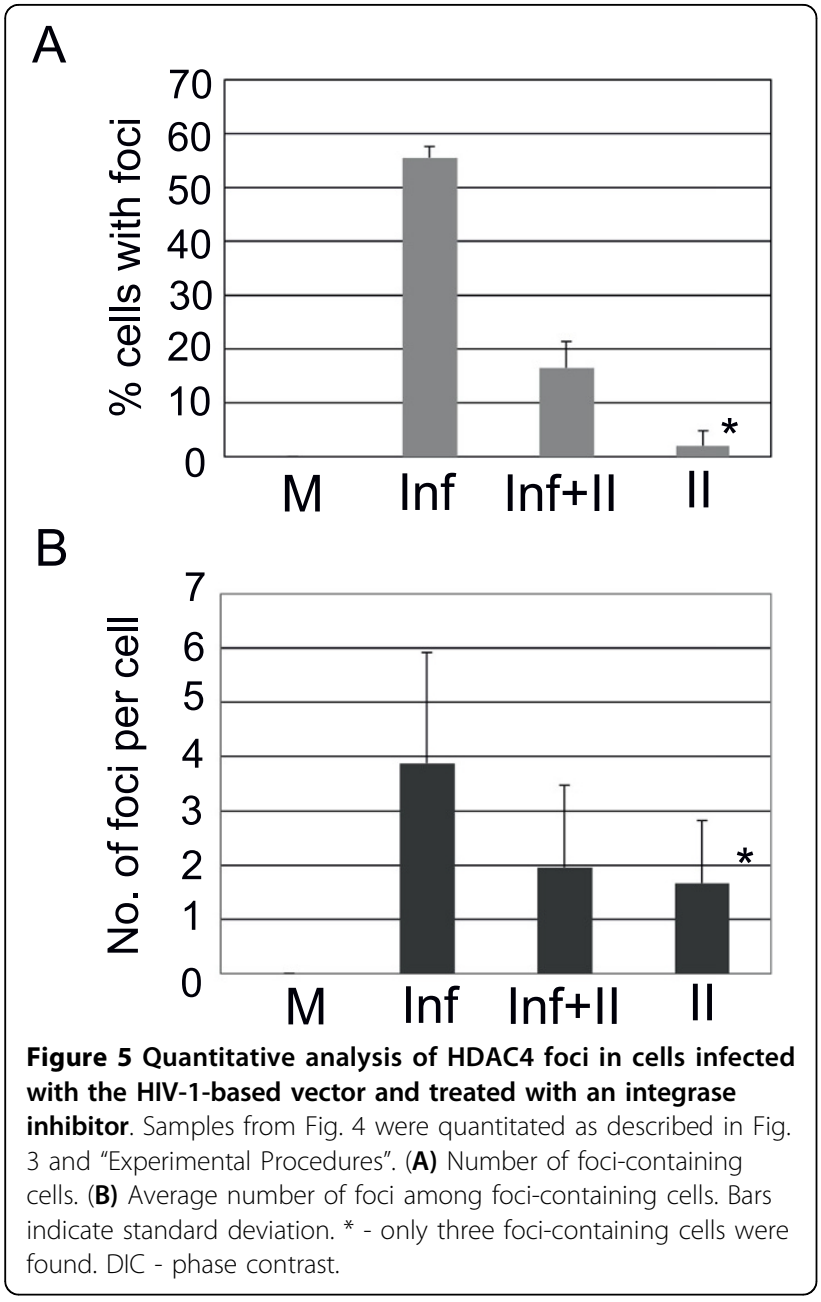

the nuclear export signal (amino acids 1-1061) fused to the EGFP protein (HDAC4-1061) have been described, and were a generous gift from Dr. X. J. Yang of McGill University $[12,29]$. The PEGFP-C1 control plasmid expressing the EGFP protein under control of the CMV promoter was purchased from Clontech (GenBank Accession \# U55763). Plasmids were transfected into HeLa cells using the Lipofectamine ${ }^{\mathrm{Tw}} 2000$ transfection reagent (Invitrogen, cat \# 11668-027) using company protocols. Cells were infected with the HIV-1-based vector two days post-transfection.

\section{Chromatin Immunoprecipitation}

HeLa cells were infected at a multiplicity of infection (m.o.i.) 0.1 for the indicated time intervals. In some cases, an integrase inhibitor was added at the time of infection (118-D-24, NIH AIDS Reagent Program). Cells were then harvested and ChIP was performed as described [35], with the anti-HDAC4 antibody $(1 \mu \mathrm{g} /$ sample, Santa Cruz Biotechnology, cat \# sc-11418X) or anti-HDAC2 antibody $(1 \mu \mathrm{g} / \mathrm{sample}$, Abcam, cat \# ab16032) or anti-HDAC6 antibody $(1 \mu \mathrm{g} /$ sample, Santa Cruz, cat \# sc-11420). Protein-associated vector DNA was detected by real-time PCR, using primers and probes detecting HIV-1 LTR. Forward primer: 5'TGTGTGCCCGTCTGTTGTGT-3'; Reverse primer: 5'CCTGCGTCGAGAGAGCTC-3'. To quantitate the viral amplicon, a TaqMan dual 5'-6-carboxyfluorescein-and 3'-6-carboxytetramethylrhodamimine-labeled probe was used: $\quad 5^{\prime}$-(FAM)-CAGTGGCGCCCGAACAGGGA(TAMRA)-3' (Integrated DNA Technologies). Real-time PCR was performed using a LightCycler 1.5 with software 3.5.3 (Roche). Reaction mixtures contained QuantiFast Probe $2 \times$ mix (Qiagen), $100 \mathrm{nM}$ probe, and $200 \mathrm{nM}$ primers. The standard cycling conditions were $95^{\circ} \mathrm{C}-3$ min followed by 50 cycles at $95^{\circ} \mathrm{C}-3 \mathrm{~s}$ and $60^{\circ} \mathrm{C}-30 \mathrm{~s}$. Samples were run in triplicate.

\section{Immunofluorescence experiments}

HeLa cells were plated at a density of $2 \times 10^{4}$ and grown on 4-well chamber slides. The following day, the cells were infected with the HIV-based vector at m.o.i. 10 for a time course study at 4,6 , and 8 hours. In another experiment, vector and an integrase inhibitor (118-D-24, final concentration of $100 \mu \mathrm{M}$ ) or the vector only had been incubated for 8 hours prior to fixation. At the indicated time points, cells were washed in PBS and fixed by adding cold methanol-acetone (1:1 volume) at room temperature for $2 \mathrm{~min}$. The slides were incubated with the primary antibody in KB buffer overnight at $4^{\circ} \mathrm{C}$. As a control, we used samples incubated in $\mathrm{KB}$ buffer with no primary antibody. The primary antibody was the rabbit polyclonal anti-HDAC4 (see above), diluted 1:500 in KB buffer. The secondary antibody, Alexa Fluor 488 donkey anti-rabbit (Invitrogen, cat \# A21206) was used at a 1:1000 dilution. Cells were then washed with PBS containing $0.1 \%$ Triton. Cells were incubated in the secondary antibodies for $1 \mathrm{hr}$ at room temperature. Cells were then stained directly with 4',6diamidino-2-phenylindole, dihydrochloride (DAPI) (Invitrogen, cat \# D1306) for 5 min at room temperature. The stained cells were washed with KB buffer and mounted with prolong gold anti-fade (Invitrogen, cat \# P36930). Images of stained cells were taken using a Nikon Eclipse TE-2000 S with fluorescence optics at an objective magnification of $20 \times$.

\section{Quantitation of HDAC4 foci in infected cells}

Random images of HeLa cells stained as described above were taken using Nikon Eclipse TE-2000 S at a magnification of $20 \times$. All cells were then counted on a randomly selected slide, both to determine the number of cells containing foci and number of foci per cell, if the cell contained foci. This had been performed in duplicate, each time on a different slide. 
A

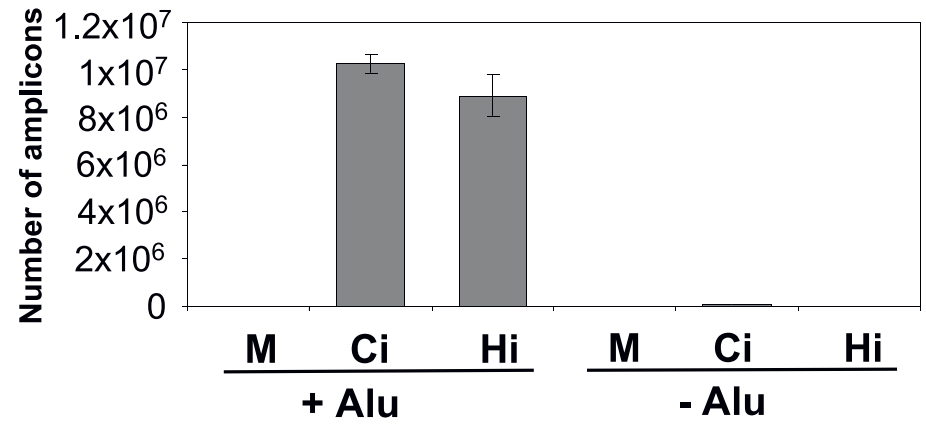

B

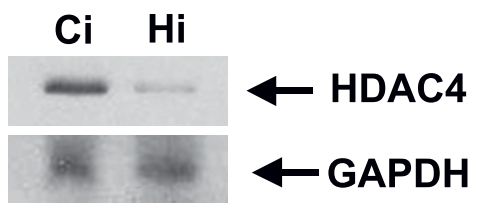

Figure 6 Effect of HDAC4 knockdown on the efficiency of integration and PIR. (A) HDAC4 was suppressed in HeLa cells using an siRNA treatment two days in a row (see "Experimental Procedures"). Two days after the first siRNA transfection, cells were infected with the HIV-1based vector. DNA was extracted 3 days post-infection and analyzed by Alu-PCR (see "Experimental Procedures"). +Alu - DNA was analyzed using Alu-PCR, - Alu - a negative control, the Alu primer was left out in the first round of PCR. M - uninfected cells, Ci - cells transfected with control siRNA and infected with the vector, Hi - cells transfected with HDAC4 siRNA and infected with the vector. (B) HDAC4 levels in cells transfected with control (Ci) and HDAC4 siRNA (Hi).

A

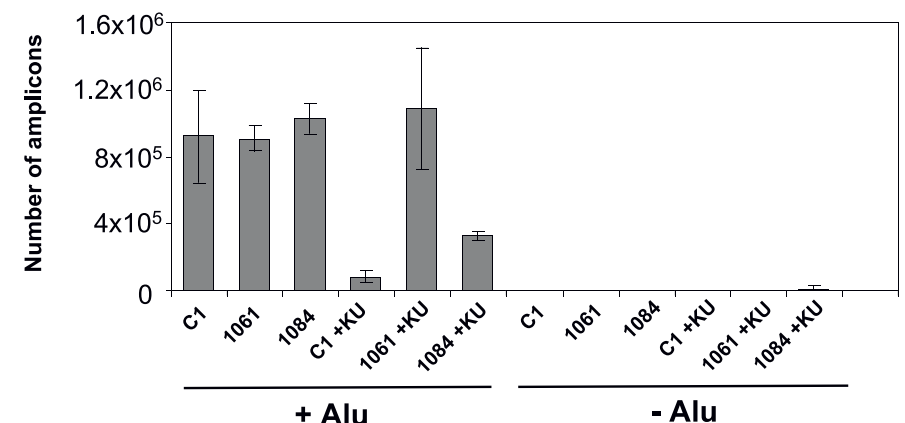

B

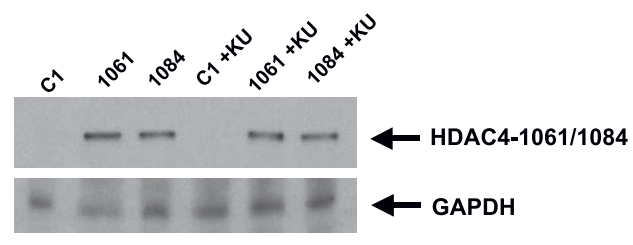

Figure 7 Effects of overexpression of HDAC4 mutants on stable integration in ATM-deficient cells. (A) Control HeLa cells and HeLa cells overexpressing HDAC4 mutants were infected and treated with the ATM inhibitor. One day post-infection, cells were harvested, DNA extracted and stable integration analyzed by Alu-PCR. C1 - cells transfected with the control EGFP expressing PEGFP-C1 plasmid and infected with the vector, 1061 - cells expressing the HDAC4-1061 mutant and infected with the vector, 1084 - cells expressing the HDAC4-1084 protein and infected with the vector. KU - the ATM inhibitor, KU-55933. +Alu - DNA was analyzed using Alu-PCR, -Alu - a negative control, the Alu primer was left out in the first round of PCR. (B) Comparison of the levels of overexpressed HDAC4 proteins. Western blotting was performed with an anti-GFP antibody (sc-9996, Santa Cruz), since HDAC4-1061 and HDAC4-1084 are fused to the GFP protein [29]. 


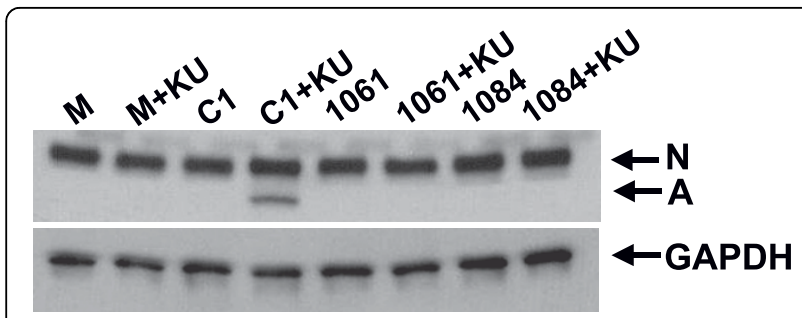

Figure 8 Effects of overexpression of HDAC4 mutants on integrase-dependent apoptosis in ATM-deficient cells. Control HeLa cells and HeLa cells overexpressing HDAC4 mutants were infected at m.o.i. 2 and treated with the ATM inhibitor $(10 \mu \mathrm{M})$. One day post-infection, cells were harvested, lysed and cell lysates subjected to western blotting with an anti-PARP antibody. M uninfected HeLa cells, C1 - cells transfected with the control EGFP expressing PEGFP-C1 plasmid and infected with the vector, 1061 cells expressing the HDAC4-1061 mutant and infected with the vector, 1084 - cells expressing the HDAC4-1084 protein and infected with the vector. $\mathrm{KU}$ - the ATM inhibitor. $\mathrm{N}$ - normal PARP protein, A - the $85-\mathrm{kDa}$ fragment of PARP, which is an apoptotic marker. GAPDH served as a loading control.

\section{Alu-PCR}

To detect and quantify fully integrated proviral DNA, a two-step nested Alu-PCR technique was conducted. Cells were infected with the HIV-1-based vector at $\mathrm{m}$. o.i. 0.1. Three days post-infection genomic DNA was extracted (Qiagen, cat \# 51306). The first round of Alu-PCR employed a primer targeting the cellular Alu sequence 5' - GCCTCCCAAAGTGCTGGGATTACAG - $3^{\prime}$ as well as the primer targeting the HIV-1 LTR/gag region, 5' - TTTTGGCGTACTCACCAGTCG - 3'. This initial amplification step used $100 \mathrm{ng}$ of genomic DNA as template. Samples were subjected to $30 \mathrm{PCR}$ cycles of $95^{\circ} \mathrm{C}-30 \mathrm{~s}, 60^{\circ} \mathrm{C}-45 \mathrm{~s}$, and $72^{\circ} \mathrm{C}-5 \mathrm{~min}$, and after the final round, samples were kept at $72^{\circ} \mathrm{C}$ for $10 \mathrm{~min}$. Products of the first round $(4 \mu \mathrm{l}$ of the 50 $\mu$ first round reaction) were used in the second, realtime $P C R$ reaction as described above (see ChIP experiments).

\section{HDAC4 siRNA-mediated knockdown}

A pool of siRNAs targeting HDAC4 (cat \# M-00349703) and a pool of non-targeting, control siRNA (cat \# D-001206-14-05) were obtained from Dharmacon. A day after plating $10^{5} \mathrm{HeLa}$ cells per $60 \mathrm{~mm}$ dish, cells were transfected with siRNA using Lipofectamine ${ }^{\text {тм }}$ RNAiMAX Transfection Reagent (Invitrogen, cat \# 13778-075) according to the manufacturer's protocol. The following day, medium was replaced, and cells were transfected again the same way. The next day (three days after cells were plated) cells were infected and assayed for integration, see $A l u$-PCR methods above. HDAC4 levels were measured three days after plating by western blotting with an anti-HDAC4 antibody (cat \# sc-11418, Santa Cruz Biotechnology).

\section{Detection of apoptosis by western blotting}

HeLa cells (transfected with either a control C1, HDAC4-1061 or HDAC4-1084 plasmid two days prior to infection, see above) were infected at mo.i. 2. KU55933 (Calbiochem, cat \# 118500-2 MG) was added at the time of infection to a final concentration of $10 \mu \mathrm{M}$. One day post-infection, cells were harvested, lysed and cell lysates subjected to western blotting with an antiPARP antibody (sc-7150, Santa Cruz Biotechnology).

\section{Acknowledgements}

This work has been supported by NIH grants CA125272 and CA135214 to R. D. and CA107956 to G.D.K.

\section{Author details}

'Division of Infectious Diseases - Center for Human Virology, Department of Medicine, Thomas Jefferson University, Philadelphia, PA 19107, USA. ${ }^{2}$ Department of Radiation Oncology, University of Pennsylvania School of Medicine, Philadelphia, PA 19104, USA. ${ }^{3}$ Center for Stem Cell Biology and Regenerative Medicine, Thomas Jefferson University, Philadelphia, PA 19107, USA. ${ }^{4}$ Kimmel Cancer Center, Immunology Program, Thomas Jefferson University, Philadelphia, PA 19107, USA.

\section{Authors' contributions}

JAS carried out the HIV-1 transduction experiments and real-time PCR-based assays. JY carried out the immunofluorescence experiments. RD wrote the manuscript and participated in western blotting and ChIP experiments GDK participated in immunofluorescence and transduction experiments. All authors read and approved the final manuscript.

\section{Competing interests}

The authors declare that they have no competing interests.

Received: 1 July 2010 Accepted: 16 September 2010 Published: 16 September 2010

\section{References}

1. Hess-Stumpp $\mathrm{H}$ : Histone deacetylase inhibitors and cancer: from cell biology to the clinic. Eur J Cell Biol 2005, 84:109-121.

2. Buggy JJ, Sideris ML, Mak P, Lorimer DD, Mclntosh B, Clark JM: Cloning and characterization of a novel human histone deacetylase, HDAC8. Biochem J 2000, 350(Pt 1):199-205.

3. Dangond F, Hafler DA, Tong JK, Randall J, Kojima R, Utku N, Gullans SR: Differential display cloning of a novel human histone deacetylase (HDAC3) cDNA from PHA-activated immune cells. Biochem Biophys Res Commun 1998, 242:648-652.

4. Emiliani S, Fischle W, Van Lint C, Al-Abed Y, Verdin E: Characterization of a human RPD3 ortholog, HDAC3. Proc Natl Acad Sci USA 1998, 95:2795-2800.

5. Hu E, Chen Z, Fredrickson T, Zhu Y, Kirkpatrick R, Zhang GF, Johanson K, Sung CM, Liu R, Winkler J: Cloning and characterization of a novel human class I histone deacetylase that functions as a transcription repressor. J Biol Chem 2000, 275:15254-15264.

6. Yang WM, Inouye C, Zeng $Y$, Bearss $D$, Seto E: Transcriptional repression by YY1 is mediated by interaction with a mammalian homolog of the yeast global regulator RPD3. Proc Natl Acad Sci USA 1996, 93:12845-12850.

7. Fischle W, Emiliani S, Hendzel MJ, Nagase T, Nomura N, Voelter W, Verdin E: A new family of human histone deacetylases related to Saccharomyces cerevisiae HDA1p. J Biol Chem 1999, 274:11713-11720.

8. Grozinger CM, Hassig CA, Schreiber SL: Three proteins define a class of human histone deacetylases related to yeast Hda1p. Proc Natl Acad Sci USA 1999, 96:4868-4873. 
9. Kao HY, Downes M, Ordentlich P, Evans RM: Isolation of a novel histone deacetylase reveals that class I and class II deacetylases promote SMRTmediated repression. Genes Dev 2000, 14:55-66.

10. Miska EA, Karlsson C, Langley E, Nielsen SJ, Pines J, Kouzarides T: HDAC4 deacetylase associates with and represses the MEF2 transcription factor. EMBO J 1999, 18:5099-5107.

11. Rundlett SE, Carmen AA, Kobayashi R, Bavykin S, Turner BM, Grunstein M: HDA1 and RPD3 are members of distinct yeast histone deacetylase complexes that regulate silencing and transcription. Proc Natl Acad Sci USA 1996, 93:14503-14508.

12. Wang $A H$, Bertos NR, Vezmar M, Pelletier $N$, Crosato M, Heng HH, Th'ng J, Han J, Yang XJ: HDAC4, a human histone deacetylase related to yeast HDA1, is a transcriptional corepressor. Mol Cell Biol 1999, 19:7816-7827.

13. de Ruijter AJ, van Gennip AH, Caron HN, Kemp S, van Kuilenburg AB: Histone deacetylases (HDACs): characterization of the classical HDAC family. Biochem J 2003, 370:737-749.

14. Frye RA: Phylogenetic classification of prokaryotic and eukaryotic Sir2like proteins. Biochem Biophys Res Commun 2000, 273:793-798.

15. Landry J, Sutton A, Tafrov ST, Heller RC, Stebbins J, Pillus L, Sternglanz R: The silencing protein SIR2 and its homologs are NAD-dependent protein deacetylases. Proc Natl Acad Sci USA 2000, 97:5807-5811.

16. Gregoretti IV, Lee YM, Goodson HV: Molecular evolution of the histone deacetylase family: functional implications of phylogenetic analysis. $J$ Mol Biol 2004, 338:17-31.

17. Kao GD, McKenna WG, Guenther MG, Muschel RJ, Lazar MA, Yen TJ: Histone deacetylase 4 interacts with $53 \mathrm{BP} 1$ to mediate the DNA damage response. J Cell Biol 2003, 160:1017-1027.

18. Basile $V$, Mantovani $R$, Imbriano $C$ : DNA damage promotes histone deacetylase 4 nuclear localization and repression of G2/M promoters, via p53 C-terminal lysines. J Biol Chem 2006, 281:2347-2357.

19. Geng L, Cuneo KC, Fu A, Tu T, Atadja PW, Hallahan DE: Histone deacetylase (HDAC) inhibitor LBH589 increases duration of gamma$\mathrm{H} 2 \mathrm{AX}$ foci and confines HDAC4 to the cytoplasm in irradiated non-small cell lung cancer. Cancer Res 2006, 66:11298-11304.

20. Daniel R, Katz RA, Skalka AM: A role for DNA-PK in retroviral DNA integration. Science 1999, 284:644-647.

21. Jeanson L, Subra F, Vaganay S, Hervy M, Marangoni E, Bourhis J, Mouscadet JF: Effect of Ku80 depletion on the preintegrative steps of HIV-1 replication in human cells. Virology 2002, 300:100-108.

22. Li L, Olvera JM, Yoder KE, Mitchell RS, Butler SL, Lieber M, Martin SL, Bushman FD: Role of the non-homologous DNA end joining pathway in the early steps of retroviral infection. EMBO J 2001, 20:3272-3281.

23. Smith JA, Daniel R: Following the path of the virus: the exploitation of host DNA repair mechanisms by retroviruses. ACS Chem Biol 2006, 1:217-226.

24. Greger JG, Katz RA, Ishov AM, Maul GG, Skalka AM: The cellular protein daxx interacts with avian sarcoma virus integrase and viral DNA to repress viral transcription. J Virol 2005, 79:4610-4618.

25. Daniel R, Kao G, Taganov K, Greger JG, Favorova O, Merkel G, Yen TJ, Katz RA, Skalka AM: Evidence that the retroviral DNA integration process triggers an ATR-dependent DNA damage response. Proc Natl Acad Sci USA 2003, 100:4778-4783.

26. Smith JA, Wang FX, Zhang H, Wu KJ, Williams KJ, Daniel R: Evidence that the Nijmegen breakage syndrome protein, an early sensor of doublestrand DNA breaks (DSB), is involved in HIV-1 post-integration repair by recruiting the ataxia telangiectasia-mutated kinase in a process similar to, but distinct from, cellular DSB repair. Virol J 2008, 5:11.

27. Daniel R, Katz RA, Merkel G, Hittle JC, Yen TJ, Skalka AM: Wortmannin potentiates integrase-mediated killing of lymphocytes and reduces the efficiency of stable transduction by retroviruses. Mol Cell Biol 2001, 21:1164-1172.

28. Lau A, Swinbank KM, Ahmed PS, Taylor DL, Jackson SP, Smith GC, O'Connor MJ: Suppression of HIV-1 infection by a small molecule inhibitor of the ATM kinase. Nat Cell Biol 2005, 7:493-500.

29. Liu F, Dowling M, Yang XJ, Kao GD: Caspase-mediated specific cleavage of human histone deacetylase 4. J Biol Chem 2004, 279:34537-34546.

30. Kim TW, Pettingell WH, Jung YK, Kovacs DM, Tanzi RE: Alternative cleavage of Alzheimer-associated presenilins during apoptosis by a caspase-3 family protease. Science 1997, 277:373-376.

31. Ficner R: Novel structural insights into class I and II histone deacetylases. Curr Top Med Chem 2009, 9:235-240.
32. Schroder AR, Shinn P, Chen H, Berry C, Ecker JR, Bushman F: HIV-1 integration in the human genome favors active genes and local hotspots. Cell 2002, 110:521-529.

33. Naldini L, Blomer U, Gallay P, Ory D, Mulligan R, Gage FH, Verma IM, Trono D: In vivo gene delivery and stable transduction of nondividing cells by a lentiviral vector. Science 1996, 272:263-267.

34. Daniel R, Greger JG, Katz RA, Taganov KD, Wu X, Kappes JC, Skalka AM: Evidence that stable retroviral transduction and cell survival following DNA integration depend on components of the nonhomologous end joining repair pathway. J Virol 2004, 78:8573-8581.

35. Smith JA, Ndoye AM, Geary K, Lisanti MP, Igoucheva O, Daniel R: A role for the Werner syndrome protein in epigenetic inactivation of the pluripotency factor Oct4. Aging Cell 2010, 9:580-591.

doi:10.1186/1743-422X-7-237

Cite this article as: Smith et al:: A role for the histone deacetylase HDAC4 in the life-cycle of HIV-1-based vectors. Virology Journal 2010 7:237.

\section{Submit your next manuscript to BioMed Central and take full advantage of:}

- Convenient online submission

- Thorough peer review

- No space constraints or color figure charges

- Immediate publication on acceptance

- Inclusion in PubMed, CAS, Scopus and Google Scholar

- Research which is freely available for redistribution

Submit your manuscript at www.biomedcentral.com/submit
Ciomed Central 